\title{
Spatial and temporal resolutions pixel level performance analysis of the onboard remote sensing electro-optical systems
}

\section{H. M. El-Sheikh}

\author{
Y. G. Yakushenkov \\ yakush@miigaik.ru
}

Moscow State University of Geodesy and Cartography (MIIGAiK), Moscow

Moscow State University of Geodesy and Cartography (MIIGAiK), Moscow

Formulas for determination of the interconnection between the spatial resolution from perspective distortions and the temporal resolution of the onboard electro-optical system for remote sensing application for a variety of scene viewing modes is offered. These dependences can be compared with the user's requirements, upon the permission values of the design parameters of the modern main units of the electro-optical system is discussed.

[DOI: http://dx.doi.org/10.2971/jeos.2014.14035]

Keywords: Onboard remote sensing electro-optical systems (ORSEOS), temporal resolution, spatial resolution

\section{INTRODUCTION}

Selecting the operational geometrical-optical scanning scheme of the ORSEOS that mounted on the flying vehicle depends upon the required task, which is closely associated with the spatial, temporal, energetic and spectral resolutions of that ORSEOS. Hence, it is important to match between the operational scanning scheme and the predetermined users requirements with the possibilities of the modern ORSEOS basic parts, first and foremost, with the parameters of optical system (lens) and focal plane array (FPA). In [1, 2], some principles for selecting the ORSEOS operational scanning schemes are explained, the determination the main design parameters and characteristics of the infrared imaging systems for remote sensing applications are discussed, and also, provide some possible ways for choosing and evaluating the ORSEOS basic parts building structure and their corresponding design parameters and characteristics.

This paper is devoted to make a relation between the spatial resolution (defined as the linear size $l_{x}$ of the projected detector element onto the scene) and the temporal resolution (defined as the dwell time $\tau_{d o}$ taken to scan across their corresponding spatial), and to explain the effect of the perspective distortion, arisen from the oblique views operating modes, upon the ORSEOS spatial and temporal resolutions.

The acquisition of oblique photography by the manned and unmanned flying platform is an area of strong development. There is a strong movement towards combining traditional nadir (vertical) images with oblique images acquired at high angles. Currently, the systematic oblique photography using multiple ORSEOSs is the much interest. To understand how these resolutions depends upon the operation mission parameters including, the flying velocity $V_{y}$, the flight height $H$ and the ORSEOS look angle $\Omega_{x}^{\prime}$ and $\Omega_{y}^{\prime}$; it is beneficial to exam- ine these resolutions in the vertical, side oblique and forward oblique operational viewing modes.

\section{DISCUSSION}

The generic operational geometrical-optical scheme of the ORSEOS in the vertical, side oblique and forward oblique viewing modes is illustrated in Figure 1. One of the ways to achieve this operational scheme is that, a flying vehicle $\mathbf{1}$ carries the ORSEOS $\mathbf{2}$ over the scene $\mathbf{3}$ and flies along the scene in the $y$ direction with a velocity $V_{y}$ at the object plane, while the ORSEOS, at the same time, viewing across the scene 3in the $x$ direction by the means of the optic-mechanical or electronic scanning with a velocity $v_{x}$ (for example, by the aid of the multi-element FPA 4).

The ORSEOS views the underlying surface (scene) 3 of dimensions $L_{x} \times L_{y}$ from a height $H$ by the means of the multielement FPA 4 of an angular size $2 \Omega_{x} \times 2 \Omega_{y}$ (nadir field of view FOV), where the FPA is located at the focal plane of the ORSEOS lens 5 with a focal length $f$. The FPA 4 may formatted in $N_{x}$ individual photosensitive elements (pixels) 6 operating in $x$ direction, or may include a format area having $N_{x} \times N_{y}$ pixels arranged in columns and rows (lines), where $N_{x}$ is the number of pixels operating in $x$ direction and $N_{y}$ is the number of pixels operating in $y$ direction. For a full FPA, $100 \%$ fill factor, the detectors are contiguous, and the pixel pitch $d_{x}$ and $d_{y}$ in the $x, y$ direction, respectively, defining the detector active dimensions, while there is no gaps between the detector elements. The IFOV angles $2 \omega_{x_{0}}$ and $2 \omega_{y_{0}}$ are the angle subtended by the $d_{x}$ and $d_{y}$ in the $x, y$ direction, respectively, while, the FOV angles $2 \Omega_{x}$ and $2 \Omega_{y}$ are the total angle subtended by the FPA size $d_{x-a r}$ and $d_{y-a r}$ in the $x, y$ direction, respectively. In the vertical viewing mode (Figure 1(a)), 
the ORSEOSs optical axis 7 is perpendicular to the FPA 4 surface where the optical axis coincides on the plumb line 8 . The FPA array 4 or the detector element (pixel) 6 is projected onto the scene 3 surface by the system optic (lens) 5 without any perspective distortion, the fundamental spatial resolution $l_{x \mathrm{o}}$ and $l_{y_{0}}$ represent the minimum linear sizes of the projected detector elements (pixels) 9 onto the scene in the $x, y$ direction, respectively. In the side oblique mode (Figure 1(b)), the ORSEOSs optical axis 7 is also perpendicular to the FPA 4 surface while it is deviated by an arbitrary look angle $\Omega_{x}^{\prime}$ in the $x$ direction relative to the plumb line 8 . The values $l_{x i}$ and $l_{y i}$ defining the $i^{\text {th }}$ spatial resolution in the $x, y$ direction, respectively, of each projected pixel 9 onto the scene 3 within the FPA 4 format array. The angles $\theta_{i}$ representing the angles within the $2 \Omega_{x}$ that measured to the center of each column within FPA 4 format and the signs $(+,-)$ determining the column location with respect to the optical axis 7 . In the forward oblique mode (Figure 1(c)), again, the ORSEOS's optical axis 7 is perpendicular to the FPA 4 surface but it is deviated by an arbitrary look angle $\Omega_{y}^{\prime}$ in the $y$ direction relative to the plumb line 8. The values $l_{x j}$ and $l_{y j}$ representing the $j^{\text {th }}$ spatial resolution in the $x, y$ direction, respectively, of each projected pixel 9 onto the scene 3 within the FPA 4 format array. The angles $\theta_{j}$ defining the angles within the $2 \Omega_{y}$ that measured to the center of each row within FPA format and the signs $(+,-)$ indicate the row location with respect to the optical axis 7.The line of sight LOS passes from the center of the projected pixel 9 through the lens nodal point 5 to the center of its corresponding pixel 6 within the FPA format array.

The FPA is limited by the array dimensions $d_{x-a r}$ and $d_{y-a r}$ in the $x, y$ direction, respectively,

$$
d_{x-a r}=N_{x} d_{x}, \quad d_{y-a r}=N_{y} d_{y}
$$

The angles $2 \Omega_{x}, 2 \Omega_{y}, 2 \omega_{x_{0}}$ and $2 \omega_{y_{0}}$ are specified by the FPA 4 geometry and the focal length $f^{\prime}$ of the lens 5 ,

$$
2 \Omega_{x}=2 \tan ^{-1}\left(N_{x} d_{x} / 2 f^{\prime}\right), \quad 2 \Omega_{y}=2 \tan ^{-1}\left(N_{y} d_{y} / 2 f^{\prime}\right)
$$

In the nadir view, i.e. $\Omega^{\prime}=\Omega_{y}^{\prime}=0$, the spatial resolution $l_{x \mathrm{o}}$ and $l_{y_{0}}$ not vary with respect to the pixels positions within the FPA 4 format array. These values can be obtained by

$$
l_{x_{\mathrm{o}}}=2 H \tan \omega_{x_{\mathrm{o}}} \approx 2 H \omega_{x_{\mathrm{o}}}, \quad l_{y_{\mathrm{o}}}=2 H \tan \omega_{y_{\mathrm{o}}} \approx 2 H \omega_{y_{\mathrm{o}}}
$$

In the case that $d x \approx 2 \omega_{x_{0}} f^{\prime}$ and $d y \approx 2 \omega y \mathrm{o} f^{\prime}$, then $l_{x_{\mathrm{o}}}$ and $l_{y_{0}}$ can be formulated as

$$
l_{x \mathrm{o}} \approx d_{x} H / f^{\prime}, \quad l_{y_{\mathrm{o}}} \approx d_{y} H / f^{\prime}
$$

In the side oblique view, i.e. $\Omega_{y}^{\prime}=0$ and $\Omega_{x}^{\prime} \neq 0$, the spatial resolution $l_{x i}$ and $l_{y i}$ vary with respect to the pixels positions within the FPA 4 format array. The $i^{\text {th }}$ spatial resolution values $l_{x i}$ and $l_{y i}$ can be determined by $[3,4]$

$$
\begin{aligned}
& l_{x i} \approx d_{x} H\left(\cos ^{2} \theta_{i}\right) / f^{\prime} \cos ^{2}\left(\Omega_{x}^{\prime} \pm \theta_{i}\right) \\
& l_{y i} \approx d_{y} H\left(\cos \theta_{i}\right) / f^{\prime} \cos \left(\Omega_{x}^{\prime} \pm \theta_{i}\right)
\end{aligned}
$$

where $\theta_{i}=\tan ^{-1}\left(i d_{x} / f^{\prime}\right)$ for $i=0,1,2, \ldots \ldots, N_{x} / 2$ and $\theta_{i}, \Omega_{x}^{\prime} \ldots$. in degree.
In the forward oblique view, i.e. $\Omega_{x}^{\prime}=0$ and $\Omega_{y}^{\prime} \neq 0$, the spatial resolution $l_{x j}$ and $l_{y j}$ vary with respect to the pixels positions within the FPA 4 format array. The $j^{\text {th }}$ spatial resolution values $l_{x j}$ and $l_{y j}$ can be obtained by $[3,4]$

$$
\begin{aligned}
& l_{x j} \approx d_{x} H\left(\cos \theta_{j}\right) / f^{\prime} \cos \left(\Omega_{y}^{\prime} \pm \theta_{j}\right) \\
& l_{y j} \approx d_{y} H\left(\cos ^{2} \theta_{j}\right) / f^{\prime} \cos ^{2}\left(\Omega_{y}^{\prime} \pm \theta_{j}\right)
\end{aligned}
$$

where $\theta_{j}=\tan ^{-1}\left(j d_{y} / f^{\prime}\right)$ for $j=0,1,2, \ldots \ldots, N_{y} / 2$ and $\theta_{j}, \Omega_{y}^{\prime} \ldots$ in degree.

The dwell time across each pixel in the $y$ direction in the aforementioned viewing modes can be determined by [5]-[8] for vertical viewing mode,

$$
T_{c o} \approx l_{y_{0}} / V_{y} \approx d_{y} /\left(V_{y} / H\right) f^{\prime},
$$

for side oblique viewing mode,

$$
T_{c i} \approx l_{y i} / V_{y} \approx d_{y}\left(\cos \theta_{i}\right) /\left(V_{y} / H\right) f^{\prime} \cos \left(\Omega_{x}^{\prime} \pm \theta_{i}\right),
$$

and for forward oblique viewing mode,

$$
T_{c j} \approx l_{y j} / V_{y} \approx d_{y}\left(\cos ^{2} \theta_{j}\right) /\left(V_{y} / H\right) f^{\prime} \cos ^{2}\left(\Omega_{y}^{\prime} \pm \theta_{j}\right) .
$$

If the motions remain uncompensated, a greater or lesser degradation depending on the flying vehicle 1 velocity-toheight ratio $\left(V_{y} / H\right)$ in image quality (resolution) occurs. The dominant equations for the image motion compensation can be considered as the line by line (row by row) scanning rate, $f_{c o}=1 / T_{c o}, f_{c i}=1 / T_{c i}$ and $f_{c j}=1 / T_{c j}$ for the vertical, side oblique and forward oblique viewing modes, respectively.

The dwell time across each pixel, in the $x$ direction, in the aforementioned viewing modes, can be obtained by [5]-[8] for vertical viewing mode,

$$
\tau_{d \mathrm{o}} \approx \eta_{c 1} T_{c o} / N_{x} \approx \eta_{c 1} d_{y} /\left(V_{y} / H\right) f^{\prime} N_{x},
$$

for side oblique viewing mode,

$$
\begin{aligned}
\tau_{d i} & \approx \eta_{c 1} T_{c i} / N_{x} \\
& \approx \eta_{c 1} d_{y}\left(\cos \theta_{j}\right) /\left(V_{y} / H\right) f^{\prime} N_{x} \cos \left(\Omega_{x}^{\prime} \pm \theta_{i}\right),
\end{aligned}
$$

for forward oblique viewing mode,

$$
\begin{aligned}
t_{d j} & \approx \eta_{c 1} T_{c j} \\
& \approx \eta_{c 1} d_{y}\left(\cos ^{2} \theta_{j}\right) /\left(V_{y} / H\right) f^{\prime} N_{x} \cos ^{2}\left(\Omega_{y}^{\prime} \pm \theta_{j}\right),
\end{aligned}
$$

where $\eta_{c 1}$ is the scan efficiency in the $x$ direction, defining the ratio of the amount of time that the $N_{x}$ effectively sample the $2 \Omega_{x}$.

The scan velocity in the $x$ direction across each pixel in the vertical, side and forward oblique mode, can be determined by, for vertical viewing mode,

$$
v_{x \mathrm{o}} \approx l_{x_{\mathrm{o}}} / \tau_{d_{\mathrm{o}}} \approx\left(V_{y} / H\right) N_{x} d_{x} / \eta_{c 1} d_{y},
$$

for side oblique viewing mode,

$$
\begin{aligned}
v_{x i} & \approx l_{x i} / \tau_{x i} \\
& \approx\left(V_{y} / H\right) N_{x} d_{x}\left(\cos \theta_{j}\right) / \eta_{c 1} d_{y} \cos \left(\Omega_{x}^{\prime} \pm \theta_{i}\right),
\end{aligned}
$$




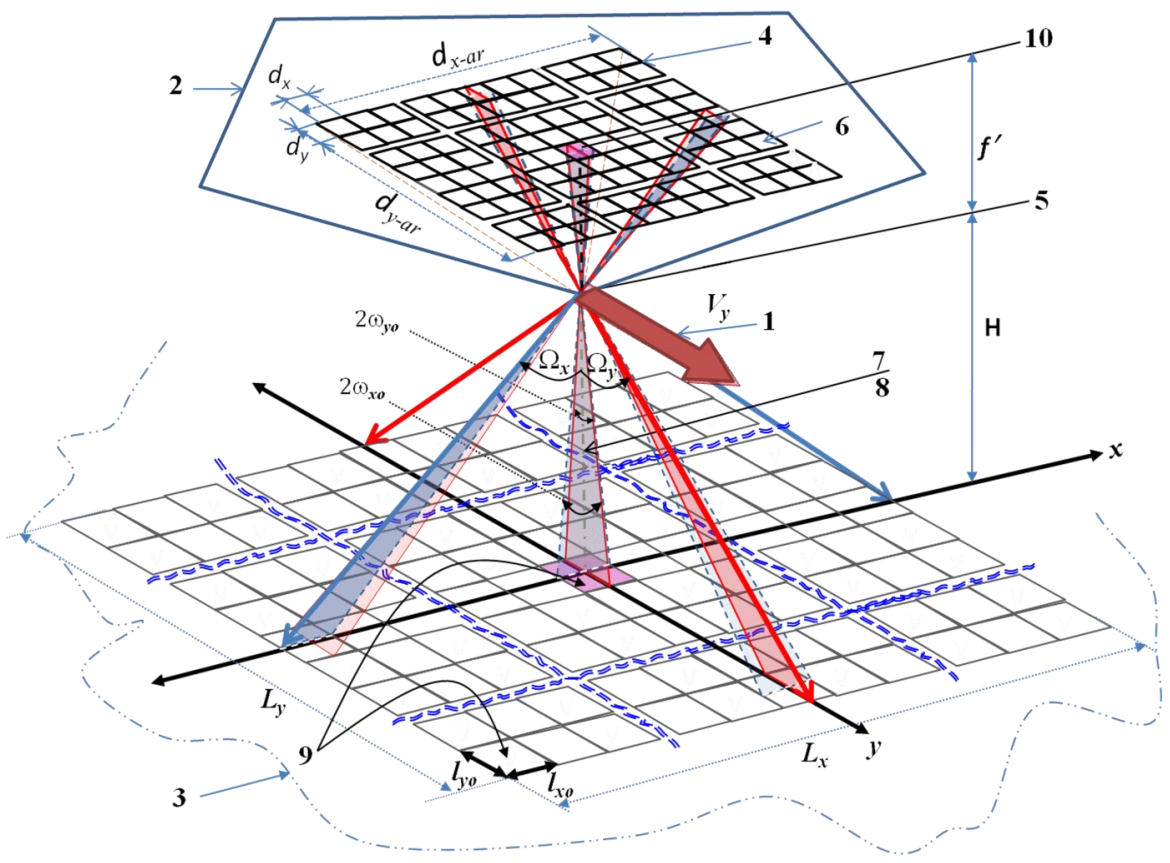

(a) An illustration of an example environment for the ORSEOS operating in a vertical viewing mode

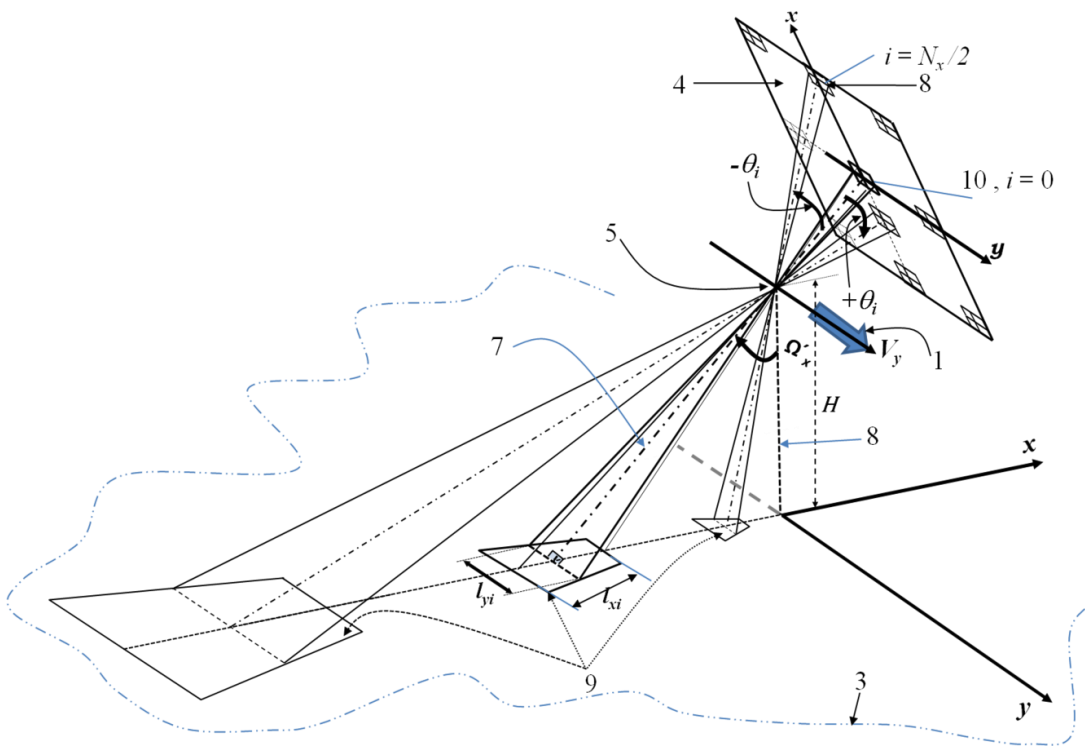

(b) An illustration of an example environment for the ORSEOS operating in a side oblique viewing mode

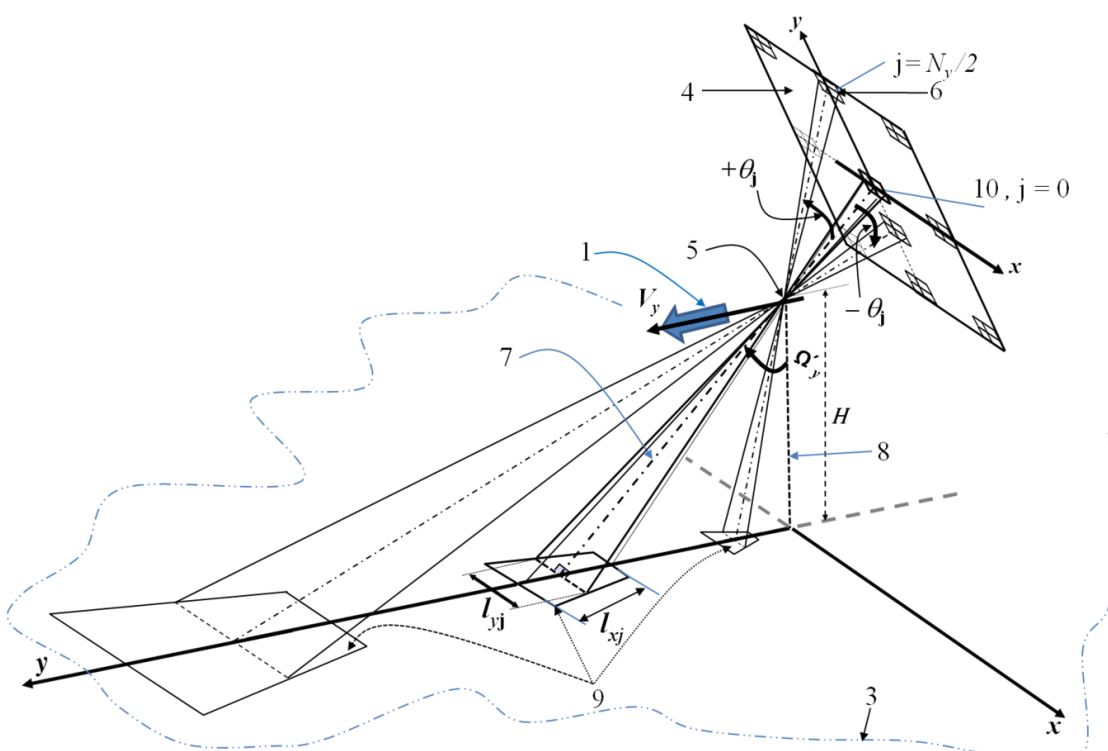

(c) An illustration of an example environment for the ORSEOS operating in a forward oblique viewing mode 
for forward oblique viewing mode

$$
v_{x j} \approx l_{x j} / \tau_{d j} \approx\left(V_{y} / H\right) N_{x} d_{x} \cos \left(\Omega_{x}^{\prime} \pm \theta_{j}\right) / \eta_{c 1} d_{y} \cos \theta_{j}
$$

The electrical (temporal) frequency of the readout circuits for each pixel is the reciprocal of its corresponding dwell time across that pixel, in the $x$ direction. These frequencies can be expressed as

$$
f_{\mathrm{o}}=1 / \tau_{d \mathrm{o}}, \quad f_{i}=1 / \tau_{d i} \text { and } f_{j}=1 / \tau_{d j},
$$

where the frequencies $f_{\mathrm{o}}, f_{i}$ and $f_{j}$ are the temporal frequency in the vertical, side oblique and forward oblique viewing modes, respectively.

The electrical bandwidth (passband) $\Delta f_{\mathrm{o}}, \Delta f_{i}$ and $\Delta f_{j}$ of the readout circuits for each pixel in the vertical, side and forward oblique mode, respectively, is derived by the consideration of response of the detector to a step-function target input. The electrical bandwidth are given by, for vertical viewing mode,

$$
\Delta f_{\mathrm{o}} \approx 1 / k_{\Delta f} \tau_{d \mathrm{o}} \approx\left(V_{y} / H\right) f^{\prime} N_{x} / k_{\Delta f} \eta_{c 1} d_{y}
$$

for side oblique viewing mode,

$$
\begin{aligned}
\Delta f_{i} & \approx 1 / k_{\Delta f} \tau_{d i} \\
& \approx\left(V_{y} / H\right) f^{\prime} N_{x} \cos \left(\Omega_{y}^{\prime} \pm \theta_{j}\right) / k_{\Delta f} \eta_{c 1} d_{y} \cos \theta_{j},
\end{aligned}
$$

and for forward oblique viewing mode,

$$
\begin{aligned}
\Delta f_{j} & \approx 1 / k_{\Delta f} \tau_{d j} \\
& \approx\left(V_{y} / H\right) f^{\prime} N_{x} \cos ^{2}\left(\Omega_{y}^{\prime} \pm \theta_{j}\right) / k_{\Delta f} \eta_{c 1} d_{y} \cos ^{2} \theta_{j},
\end{aligned}
$$

where the factor $k \Delta f \approx 0.5$ to 2 depending on the application (discrimination criteria) [2]. For example $k \Delta f \approx 2$ is used for detection system.

For the side oblique perspective, the $i^{\text {th }}$ distortion coefficients $k_{x i}$ and $k_{y i}$ in the $x, y$ direction, respectively, can be determined by

$$
\begin{gathered}
k_{x i}=l_{x i} / l_{x \mathrm{o}}=\left(\cos ^{2} \theta_{i}\right) / \cos ^{2}\left(\Omega_{x}^{\prime} \pm \theta_{i}\right) \\
k_{y i}=l_{y i} / l_{y \mathrm{o}}=\left(\cos \theta_{i}\right) / \cos \left(\Omega_{x}^{\prime} \pm \theta_{i}\right) .
\end{gathered}
$$

Similarly for the forward oblique perspective, the $j^{\text {th }}$ distortion coefficients $k_{x j}$ and $k_{y j}$ in the $x, y$ direction, can be achieved by

$$
\begin{gathered}
k_{x j}=l_{x j} / l_{x \mathrm{o}}=\left(\cos \theta_{j}\right) / \cos \left(\Omega_{y}^{\prime} \pm \theta_{j}\right) \\
k_{y j}=l_{y j} / l_{y \mathrm{o}}=\left(\cos ^{2} \theta_{i}\right) / \cos ^{2}\left(\Omega_{y}^{\prime} \pm \theta_{i}\right) .
\end{gathered}
$$

The spatial resolution values are mainly dependent upon the perspective distortion which arising due to the orientation of the ORSEOS's optical axis 7 relative to relative to the plumb line 8 during the acquisition process. For the oblique views, the distorted projected pixels 9 are gathering together to construct the whole distorted image of the scene 3 .

The fundamental (dominant) frequency of interest $f_{\mathrm{o}}$ is the main parameter for designing the detector readout circuits. The frequency $f_{\mathrm{o}}$ is the higher electrical frequency of the readout circuits.
The temporal resolution is directly related to its IFOV and the probability of scan (or sample) the entire field in a given time, the scanning period (frame time) $T_{\mathrm{ko}}$. The ratio of the period of time it takes to scan the field to the total frame time $T_{\mathrm{ko}}$ is sometimes referred to as the scanning efficiency factor $\eta$ in another words, $\eta$ is the ratio of the amount of time that the $N_{\text {total }}=N_{x} \times N_{y}$ effectively sample the whole FOV to the total time $T_{\text {ko }}$ taken for one frame. The frame to frame times $T_{\mathrm{ko}}$ for the detector array are determined by the array dimension $N_{y} d_{y}$ in the flight direction, the velocity-to-height ratio $\left(V_{y} / H\right)$, the lens focal length $f^{\prime}$, the ORSEOS look angles $\left(\Omega_{x}^{\prime}, \Omega_{y}^{\prime}\right)$, in the vertical, side and forward oblique mode, can be determined by, the scanning efficiency factor $\eta$, the scan efficiency $\eta_{1}$ in the $x$ direction and the degree of overlap (OL) desired between frames. The equations governing frame to frame time $T_{\mathrm{ko}}, T_{k}$ and $T_{k}^{\prime}$ in the vertical, side oblique and forward oblique viewing modes, respectively, can be expressed as the following: for vertical viewing mode,

$$
T_{\mathrm{ko}} \approx \eta_{c 1} N_{y} d_{y}(1-\mathrm{OL}) / \eta_{c}\left(V_{y} / H\right) f^{\prime},
$$

for side oblique viewing mode,

$$
T_{k} \approx \eta_{c 1} N_{y} d_{y}(1-\mathrm{OL}) / \eta_{c}\left(V_{y} / H\right) f^{\prime} \cos \Omega_{x}^{\prime} \approx T_{\mathrm{ko}} k_{y},
$$

where the distortion coefficient $k_{y}=1 / \cos \Omega_{x}^{\prime}$, for forward oblique viewing mode,

$$
T_{k}^{\prime} \approx \eta_{c 1} N_{y} d_{y}(1-\mathrm{OL}) / \eta_{c}\left(V_{y} / H\right) f^{\prime} \cos ^{2} \Omega_{y}^{\prime} \approx T_{k \mathrm{o}} k_{y}^{\prime}
$$

where the distortion coefficient $k_{y}^{\prime}=1 / \cos ^{2} \Omega_{y}^{\prime}$.

The frame rate is the reciprocal of the frame time to frame time $T_{\mathrm{ko}}, T_{\mathrm{k}}$ and $T_{\mathrm{k}}$, respectively,

$$
f_{\mathrm{ko}}=1 / T_{\mathrm{ko}}, \quad f_{\mathrm{k}}=1 / T_{\mathrm{k}} \quad \text { and } f_{\mathrm{k}}=1 / T_{\mathrm{k}},
$$

where the $f_{\mathrm{ko}}, f_{\mathrm{k}}$ and $f \mathrm{k}$ are the frame rate in the vertical, side oblique and forward oblique viewing modes, respectively.

The parameters of the spatial and temporal resolution of the ORSEOS in the aforementioned viewing modes can be tabulated as in Table 1.

The requirements of these coefficient values may be very important. Figure 2 illustrates the dependence of the perspective distortion upon the different angular position values of the $\operatorname{LOS} \Omega_{x}^{\prime}$ and $\Omega_{y}^{\prime}$.

Figure 2 and Table 1 can be used to determine the increasing of the linear subtense of the projected pixels onto the scene surface and their corresponding temporal resolution degraded values which arisen from the perspective distortion.

Today, it is known a large variety in the lenses that can be used with ORSEOS. As an example, for IR band 7-14 $\mu \mathrm{m}$ wavelength ranges, there are the lenses brand ASIO and STRIX with circular FOV $110^{\circ}$, focal length $7 \mathrm{~mm}$, relative aperture 1.4 and image diagonal $21 \mathrm{~mm}[9,10]$, these lenses can be integrated with FPA large format $1024 \times 1024$ in $17 \mu \mathrm{m}$ pixel pitch [11].

If an aircraft flies at velocity $V_{y}=36 \mathrm{~ms}^{-1}$ at altitude $H=64 \mathrm{~m}$ and carries the ORSEOS that utilizing the aforementioned IR 


\begin{tabular}{|c|c|c|c|}
\hline Item & $\begin{array}{l}\text { Vertical mode } \\
\Omega_{x}^{\prime}=\Omega_{y}^{\prime}=0^{\circ}\end{array}$ & $\begin{array}{l}\text { Side oblique mode } \\
\Omega_{y}^{\prime}=0^{\circ}, \Omega_{x}^{\prime} \leq 90^{\circ}\end{array}$ & $\begin{array}{l}\text { Forward oblique mode } \\
\Omega_{x}^{\prime}=0^{\circ}, \Omega_{y}^{\prime} \leq 90^{\circ}\end{array}$ \\
\hline $\begin{array}{l}\text { Pixel distortion coefficient } \\
\text { in } x \text { direction }\end{array}$ & $k_{x}=k_{x}^{\prime}=1$ & $k_{x i}=\frac{\cos ^{2} \theta_{i}}{\cos ^{2}\left(\Omega_{x}^{\prime} \pm \theta_{i}\right)}$ & $k_{x j}=\frac{\cos \theta_{j}}{\cos \left(\Omega_{y}^{\prime} \pm \theta_{j}\right)}$ \\
\hline $\begin{array}{l}\text { Pixel distortion coefficient } \\
\text { in } y \text { direction }\end{array}$ & $k_{y}=k_{y}^{\prime}=1$ & $k_{y i}=\frac{\cos \theta_{i}}{\cos \left(\Omega_{x}^{\prime} \pm \theta_{i}\right)}$ & $k_{y j}=\frac{\cos ^{2} \theta_{j}}{\cos ^{2}\left(\Omega_{y}^{\prime} \pm \theta_{j}\right)}$ \\
\hline $\begin{array}{l}\text { Pixel spatial resolution } \\
\text { in } x \text { direction }\end{array}$ & $l_{x \mathrm{o}} \approx d_{x} H / f^{\prime}$ & $l_{x \mathrm{i}} \approx l_{x \mathrm{o}} k_{x i}$ & $l_{x j} \approx l_{x \mathrm{o}} k_{x j}$ \\
\hline $\begin{array}{l}\text { Pixel spatial resolution } \\
\text { in } y \text { direction }\end{array}$ & $l_{y_{\mathrm{o}}} \approx d_{y} H / f^{\prime}$ & $l_{y i} \approx l_{y o} k_{y i}$ & $l_{y j} \approx l_{y \mathrm{o}} k_{y j}$ \\
\hline $\begin{array}{l}\text { Pixel dwell time } \\
\text { in } x \text { direction }\end{array}$ & $T_{c o} \approx d_{y} /\left(V_{y} H\right) f^{\prime}$ & $T_{c i} \approx T_{c o} k_{y i}$ & $T_{c j} \approx T_{c o} k_{y j}$ \\
\hline $\begin{array}{l}\text { Pixel dwell time } \\
\text { in } y \text { direction }\end{array}$ & $\tau_{d \mathrm{o}} \approx \eta_{\mathrm{c} 1} d_{y} /\left(V_{y} / H\right) f^{\prime} N_{x}$ & $\tau_{d i} \approx \tau_{d \mathrm{o}} k_{y i}$ & $\tau_{d j} \approx \tau_{d \mathrm{o}} k_{y j}$ \\
\hline $\begin{array}{l}\text { Pixel scanning rate } \\
\text { in } y \text { direction }\end{array}$ & $f_{c o} \approx\left(V_{y} / H\right) f^{\prime} / d_{y}$ & $f_{c i} \approx f_{c o} / k_{y i}$ & $f_{c j} \approx f_{c o} / k_{y j}$ \\
\hline $\begin{array}{l}\text { Scanning velocity } \\
\text { in } x \text { direction }\end{array}$ & $v_{x \mathrm{o}} \approx\left(V_{y} / H\right) N_{x} d_{x} / \eta_{\mathrm{c} 1} d_{y}$ & $v_{x i} \approx v_{x \mathrm{o}} k_{y i}$ & $v_{x j} \approx v_{x \mathrm{o}} k_{y j}$ \\
\hline $\begin{array}{l}\text { Pixel readout circuit } \\
\text { temporal frequency }\end{array}$ & $f_{\mathrm{o}} \approx\left(V_{y} / H\right) f^{\prime} N_{x} / \eta_{\mathrm{c} 1} d_{y}$ & $f_{i} \approx f_{\mathrm{o}} / k_{y i}$ & $f_{j} \approx f_{\mathrm{o}} / k_{y j}$ \\
\hline $\begin{array}{l}\text { Pixel readout circuit } \\
\text { electrical bandwidth }\end{array}$ & $\Delta f_{\mathrm{o}} \approx\left(V_{y} / H\right) f^{\prime} N_{x} / k_{\Delta f} \eta_{\mathrm{c} 1} d_{y}$ & $\Delta f_{i} \approx \Delta f_{\mathrm{o}} / k_{y i}$ & $\Delta f_{j} \approx \Delta f_{\mathrm{o}} / k_{y j}$ \\
\hline Frame to frame time & $T_{\mathrm{ko}} \approx \eta_{\mathrm{c} 1} N_{y} d_{y}(1-\mathrm{OL}) / \eta_{\mathrm{c}}\left(V_{y} / H\right) f^{\prime}$ & $\begin{array}{c}T_{\mathrm{k}} \approx T_{\mathrm{ko}} k_{y} \\
k_{y}=1 / \cos \Omega_{x}^{\prime}\end{array}$ & $\begin{array}{c}T_{\mathrm{k}}^{\prime} \approx T_{\mathrm{ko}} k_{y}^{\prime} \\
k_{y}^{\prime}=1 / \cos ^{2} \Omega_{y}^{\prime}\end{array}$ \\
\hline Frame rate & $f_{\mathrm{ko}} \approx \eta_{\mathrm{c}}\left(V_{y} / H\right) f^{\prime} / \eta_{\mathrm{c} 1} N_{y} d_{y}(1-\mathrm{OL})$ & $\begin{array}{c}f_{\mathrm{k}}=f_{\mathrm{ko}} / k_{y} \\
k_{y}=1 / \cos \Omega_{x}^{\prime}\end{array}$ & $\begin{array}{c}f_{\mathrm{k}}^{\prime}=f_{\mathrm{ko}} / k_{y}^{\prime} \\
k_{y}=1 / \cos \Omega_{x}^{\prime}\end{array}$ \\
\hline
\end{tabular}

TABLE 1 Spatial and temporal resolution parameters of the ORSEOS.

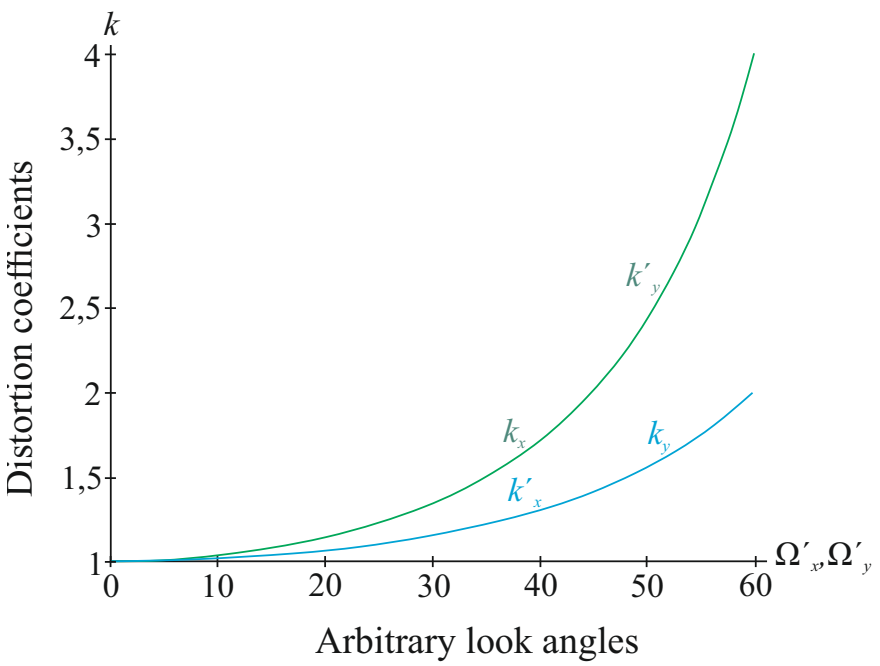

FIG. 2 Dependence of the perspective distortion upon the arbitrary looks angles $\Omega_{x}^{\prime}$

lens ASIO and the FPA large format in $17 \mu \mathrm{m}$ pixel pitch, to view the scene in the different viewing modes including, the vertical mode Figure 1(a), the side oblique mode Figure 1(b) such that the look angle $\Omega_{x}^{\prime}=55^{\circ}, \Omega_{y}^{\prime}=0^{\circ}$ and the forward oblique mode Figure 1 (c) given that $\Omega_{x}^{\prime}=0^{\circ}, \Omega_{y}^{\prime}=45^{\circ}$. In the case that, the scanning efficiency factor $\eta_{\mathrm{c}}=0.5$, the line scan efficiency $\eta_{\mathrm{c} 1}=0.9$, the factor $k \Delta f=0.5$ and the degree of overlap desired between frames $\mathrm{OL}=1$. Table 2 illustrates the spatial and temporal resolutions pixel level performance analysis at the FPA central pixel 10, which denoted by for the aforementioned example.

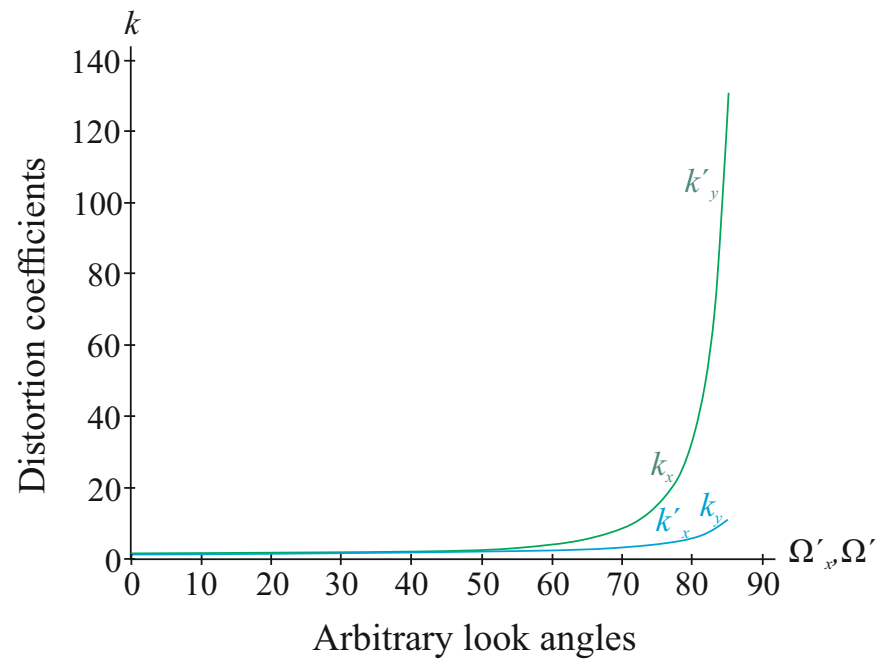

In the vertical viewing mode case, the ORSEOS will view a scene with spatial resolution values of all projected pixels $l_{x_{\mathrm{o}}} \approx 11.2 \mathrm{~cm}$ and $l_{y_{\mathrm{o}}} \approx 11.2 \mathrm{~cm}$ in the $x, y$ direction, respectively. It is notable that, as the $\Omega_{x}^{\prime}, \Omega_{y}^{\prime}$ values increase; these values will be increased which leads to decrease the ORSEOS spatial and temporal resolutions values. Then, if it is required to observe a large coverage area (scene) with high spatial and temporal resolutions without using neither the opto-mechanical scanning systems nor the step-scanning systems, it advisable to use a high resolution wide angled lens with a large format FPA in a small pixel pitch. 


\begin{tabular}{|c|c|c|c|}
\hline $\begin{array}{c}\text { Vertical mode } \\
\Omega_{x}^{\prime}=\Omega_{y}^{\prime}=0^{\circ}\end{array}$ & $\begin{array}{c}\text { Side oblique mode } \\
\Omega_{y}^{\prime}=0^{\circ}, \Omega_{x}^{\prime} \leq 90^{\circ}\end{array}$ & $\begin{array}{c}\text { Forward oblique mode } \\
\Omega_{x}^{\prime}=0^{\circ}, \Omega_{y}^{\prime} \leq 90^{\circ}\end{array}$ & Units \\
\hline$k_{x}=k_{x}^{\prime}=1$ & $k_{x i}=3.0396$ & $k_{x j}=1.4142$ & - \\
\hline$k_{y}=k_{y}=1$ & $k_{y i}=1.7434$ & $k_{y j}=2$ & - \\
\hline$l_{x \mathrm{o}} \approx 11.2$ & $l_{x i} \approx 34$ & $l_{x j} \approx 15.8$ & $\mathrm{~cm}$ \\
\hline$l_{y \mathrm{o}} \approx 11.2$ & $l_{y i} \approx 19.5$ & $l_{y j} \approx 22.4$ & $\mathrm{~cm}$ \\
\hline$T_{c o} \approx 3.103$ & $T_{c i} \approx 5.4097$ & $T_{c j} \approx 6.206$ & $\mathrm{~ms}$ \\
\hline$\tau_{d \mathrm{o}} \approx 2.7272$ & $\tau_{d i} \approx 4.7546$ & $\tau_{d j} \approx 5.4544$ & $\mu \mathrm{s}$ \\
\hline$f_{c \mathrm{o}} \approx 0.3222$ & $f_{c i} \approx 0.1848$ & $f_{c j} \approx 0.1611$ & $\mathrm{kHz}$ \\
\hline$v_{x \mathrm{o}} \approx 890.43$ & $v_{x i} \approx 1552.37$ & $v_{x j} \approx 629.63$ & $\mathrm{rad} \cdot \mathrm{s}^{-1}$ \\
\hline$f_{\mathrm{o}} \approx 0.36664$ & $f_{i} \approx 0.2103$ & $f_{j} \approx 0.18332$ & $\mathrm{MHz}$ \\
\hline$\Delta f_{\mathrm{o}} \approx 0.73328$ & $\Delta f_{i} \approx 0.4206$ & $\Delta f_{j} \approx 0.36664$ & $\mathrm{MHz}$ \\
\hline$T_{k \mathrm{o}} \approx 5.719$ & $T_{k} \approx 9.965$ & $T_{k} \approx 11.438$ & $\mathrm{~s}$ \\
\hline$f_{k \mathrm{o}} \approx 0.175$ & $f_{k}=0.1$ & $f_{k}=0.0875$ & $\mathrm{fps}$ \\
\hline
\end{tabular}

TABLE 2 Spatial and temporal resolutions performance analysis at the FPA central pixel 10 for the aforementioned example.

\section{CONCLUSION}

The deduced relations and graphs not only can help to choose the design parameters of the lens and the FPA for a given value of perspective distortion, but also serve to justify the required flying velocity and altitude for a predetermined viewing area of the underlying surface (scene) and the geometrical resolution values onto the scene surface, i.e., matching between the "internal" parameters ORSEOS (its design parameters) with the "external" parameters (user equipment requirements).

Performance evaluation of the ORSEOS depend upon the viewing modes of operation, including the vertical, side oblique and forward oblique viewing modes, that are directly affect on the ORSEOS's spatial and temporal resolution parameters and characteristics.

The velocity-to-height ratio $\left(V_{y} / H\right)$ describes the angular rate of motion of the scene with respect to the moving platform (vehicle) and hence, the resulting motion of the image in the focal plane. The $\left(V_{y} / H\right)$ is one of the dominant parameters in ORSEOS design because it determines the scan rates and the electrical signal bandwidth. The maximum value $\left(V_{y} / H\right)_{\max }$ is used to start the design trade-off process.

\section{References}

[1] Y. G. Yakushenkov, "Selecting the scanning scheme and determination of the main design parameters of the infrared remote sensing systems," J. Proc. High. Edu. Geodesy Aerial Photography 6, 118-122 (2012).
[2] Y. G. Yakushenkov, Electro-optical devices: theory and design (Logos, Moscow, 2011).

[3] V. V. Tarasov, and Y. G. Yakushenkov, Infrared imaging systems (Logos, Moscow, 2004).

[4] V. V. Tarasov, and Y. G. Yakushenkov, Dual and multi-spectral band electro-optical systems utilizing focal plane arrays (Logos, Moscow, 2007).

[5] J. S. Accetta, D. S. Shumaker, and S. B. Campana (eds.), Infrared and electro-optical systems handbook, vol. 5: passive electrooptical systems (SPIE optical engineering press, Washington, 1993).

[6] R. G. Driggers, P. Cox, and T. Edwards, Introduction to infrared and electro-optical systems (Artech house, London, 1999).

[7] A. G. Lareau, G. W. Willey, R. A. Bennett, S. R. Beran, “Electrooptical step-frame camera system with image motion compensation," EP patent 0830788 B1 (2000).

[8] B. A. Matthews, and B. H. Coon, "Electro-optical reconnaissance system with forward motion compensation," EP patent $1313308 \mathrm{~A} 2$ (2003).

[9] H. M. El-Sheikh, and Y. G. Yakushenkov, "Spatial resolution of the onboard remote sensing electro-optical system," J. Proc. High. Edu. Surveying Aerial Photography 3, 109-114 (2014).

[10] H. M. El-Sheikh, and Y. G. Yakushenkov, "Temporal resolution of the onboard remote sensing electro-optical system," J. Opt. Technol. 81, 1-5 (2014).

[11] H. Lutz, R. Breiter, S. Rutzinger, T. Schallenberg, J. Wendler, and J. Ziegler, "High-performance IR detector modules for army applications," SPIE Proc. 8704, 87040A (2013). 